\title{
Does using different entrance points for intramedullary nails affect clinical outcomes for femoral shaft fractures? A retrospective clinical comparative study
}

\author{
(iD) Mehmet Salih Soylemez, iD Tayyar Taylan Oz \\ Department of Orthopaedics and Traumatology, Umraniye Training and Research Hospital, Istanbul, Turkey
}

\begin{abstract}
OBJECTIVE: We examined the clinical and radiological outcomes of femoral shaft fractures treated with two different intramedullary nail designs using either greater trochanteric or trochanteric fossa entrance.

METHODS: The medical records of patients undergoing operations for a shaft fracture either with a nail with trochanteric entrance or trochanteric fossa entrance were retrospectively reviewed. Inclusion criteria were: having the necessary medical records including radiographic images and follow-up data, at least 12 months of follow-up, skeletal maturity ( $\geq 16$ years of age) not having osteoporosis ( $\leq 60$ years of age). Exclusion criteria were: pathological fractures, fragility fractures, fractures that extend to hip or knee joint capsule, lack of enough medical data, less than 12 months of follow up, and patients yet to reach skeletal maturity.

RESULTS: A total of 65 patients ( 67 femur fractures) were treated with intramedullary nails using a trochanteric fossa entrance (TFE) and 21 patients (23 femur fractures) were treated with nails using a greater trochanteric entrance (GTE). No statistically significant differences were evident between groups in terms of union time, blood loss, need for implant removal, implant failure, or revision operation. However, the duration of postoperative hospitalization was significantly shorter in the GTE nail group and the need for open reduction of the fracture was less common in these patients. Although there were no significant differences between groups in terms of complications and union among isthmal and infra-isthmal fractures, malreduction and iatrogenic fractures were more common with the use of GTE nails for treatment of supra-isthmal fractures.

CONCLUSION: Use of intramedullary nails via both GTE and TFE were safe and efficient for the treatment of isthmal and infra-isthmal fractures. However, varus malalignments associated with iatrogenic fractures were more common with trochanteric entrance nails. Together, our results show that the use of nails via TFE may represent a safer option for surgical treatment of supra-isthmal fractures.
\end{abstract}

Keywords: Femur shaft fracture; greater trochanter entry; trochanteric fossa entry.

Cite this article as: Soylemez MS, Oz TT. Does using different entrance points for intramedullary nails affect clinical outcomes for femoral shaft fractures? A retrospective clinical comparative study. North Clin Istanb 2020;7(6):609-618.

B eginning with the work of Gerhard Küntscher in the 1940's advocating for the use of intramedullary nails for the treatment of the femoral shaft fractures, nails have become the golden standard for surgical fixation in skeletally mature patients [1]. While both the trochanteric fossa entrance (TFE) and greater trochan- teric entrance (GTE) have been used for the placement of intramedullary nails, TFE is commonly considered the superior starting point compared to GTE in which the nail is inserted lateral to the tip of the trochanter, which could lead to varus malalignment, eccentric reaming of the medial cortex of the proximal fragment,

Received: April 10, 2020 Accepted: May 29, 2020 Online: November 27, 2020

Correspondence: Mehmet Salih SOYLEMEZ, MD. Umraniye Egitim ve Arastirma Hastanesi, Ortopedi ve Travmatoloji Klinigi, Istanbul, Turkey.

Tel: +90 2166507676 e-mail: slhsylmz@gmail.com

(c) Copyright 2020 by Istanbul Provincial Directorate of Health - Available online at www.northclinist.com 
comminution at the fracture site, or both [2]. The use of conventional nails using TFE has decreased significantly in recent years due to the increasing number of publications showing they are more likely to cause iatrogenic collum femoris fractures, and the use of new nails allowing trochanteric access has been popularized. However, other studies have reported no difference between the two nails in terms of functional outcomes and complications, suggesting that any difference may be minimal [3].

Currently, there is still debate over what is the best entry point for the intramedullary nailing of femoral shaft fractures [4]. Abductor muscles and tendons, branches of the medial circumflex femoral artery, and the hip joint capsule are at risk during antegrade nailing of shaft fractures. Moein et al. [5] investigated the risks and safety of the two entry points with respect to the adjacent soft tissues on cadaveric femurs, and found that GTE was a safer option. This suggests that clinical morbidity may result from direct soft tissue injury after nailing through the trochanteric fossa, which may be reduced by choosing the route through the greater trochanter. However, GTE may be associated with a higher risk for iatrogenic fractures, increasing the need for improved imaging for the safe and accurate insertion of laterally started nails rather than relying on anatomic landmarks to determine the entry point of laterally inserted nails, [6] which may lead to increased tension on the proximal lateral cortex and destabilization of the lateral wall. By contrast, nails inserted via GTE have been shown to decrease the duration of the surgery and ease the application of the nail, particularly in obese patients as the greater trochanter is superficially located under the skin. Entrance from this point needs less adduction of extremity, which makes collum femoris fractures less likely to occur [7-9].

We compared the clinical and radiological results of patients treated with either implant based on operative parameters, iatrogenic fractures, and union outcomes. We hypothesized that iatrogenic collum femoris fractures with use of TFE nails would be less common than anticipated. For isthmal and infra-isthmal fractures there were no differences between groups based in terms of technical demands, reduction quality, time of surgery, or complications. However, for supra-isthmal fractures there were several factors that must be assessed, which can lead to reduced quality and malalignment rather outside of nail design.

\section{MATERIALS AND METHODS}

All patients treated with long antegrade intramedullary nails between 2010-2018 in the same institution were included to the study. After obtaining institutional ethics committee approval (ID: B.10.1.TKH.4.34.H.GP.0.01/77) medical records of the patients were reviewed retrospectively. This study was conducted in accordance with principles for human experimentation as defined in the Declaration of Helsin$\mathrm{ki}$. Informed consent was obtained from all individuals prior to surgery.

Inclusion criteria were as follows: patients undergoing operations for a shaft fracture either with a nail with trochanteric entrance (TRIGEN TAN nail; Smith $\&$ Nephew, London, UK) or trochanteric fossa (TRIGEN FAN nail; Smith \& Nephew, London, UK) entrance, with the necessary medical records including radiographic images and follow-up data, at least 12 months of follow-up, skeletal maturity ( $\geq 16$ years of age) not having osteoporosis ( $\leq 60$ years of age). Exclusion criteria were as follows: pathological fractures, fragility fractures, fractures that extend to hip or knee joint capsule, surgery after complication of a previous surgery performed with a plate, surgery for a peri-implant fracture, lack of enough medical data, less than 12 months of follow up, and patients yet to reach skeletal maturity.

The choice of nail used for the surgery was at the discretion of the operating surgeon. All surgeries were performed using one of eight orthopedic trauma surgeons or under supervisions of these surgeons by six senior residents.

\section{Evaluation}

Health Information Systems (HIS V5), a digital web service, was used to obtain and review the follow-up data of the patients. $\mathrm{X}$-rays were evaluated by both authors for angular measurements and union time, using digital picture archive and communication system (PACS). Any conflict was resolved by consensus after a meeting between the two authors.

Age, sex, trauma pattern, existence of open fracture, American Society of Anesthesiologists score (ASA), and existence of accompanying fractures were evaluated for each patient. Duration of surgery, duration of postoperative stay in hospital, follow-up time, time to union, amount of blood loss, need for transfusion, need for an open reduction via a mini stoppa incision, and features 
of proximal and distal locking option performed for the patient were also reviewed.

Trauma pattern was defined as "low-energy trauma" if the fracture was sustained after a simple fall in the street or from a height less than a meter. Remaining fractures obtained with a higher energy were defined as "high-energy trauma." Duration of surgery was defined as the time between starting of the skin incision to the wound closure for shaft fracture surgery. Time of surgery for different fractures at the same session was excluded. Union of the fracture was defined as existence of callus on at least two cortices on both anterior-posterior (AP) and lateral $\mathrm{X}$-rays with pain free weight bearing during follow-ups [7]. Delayed union was defined as slow progression of fracture healing extending beyond 4 months and nonunion was defined as no progression of fracture healing after 6 months. Malalignment was defined as 10 degrees of angulation in any plane, and 15 degrees malrotation. All fractures were classified using AO/OTA [10] classification to define the extent of the fracture. They were classified as supra-isthmal, isthmal, and infra-isthmal to determine the effects of the level of the fracture on results [7]. Open fractures were classified using the Gustilo-Anderson classification [11].

\section{Treatment Protocol and Follow-ups}

All patients received low-molecular-weight heparin $(\mathrm{Cl}$ exane 4000 anti-Xa, Sanofi Aventis) starting from the first admission and continued until postoperative week 3 . All patients were followed on a skeletal traction passed from tuberositas tibiae until the surgery. Antibiotic prophylaxis ( $1 \mathrm{~g}$ cefazolin for the patients $<70 \mathrm{~kg}$ and $2 \mathrm{~g}$ cefazolin for the patients $>70 \mathrm{~kg}$ ) was started $30 \mathrm{~min}$ prior to surgery, with two additional doses administered at postoperative day 1 for the closed fractures. Use of antibiotic prophylaxis for the open fractures depended on the type of the open wound, with specific antibiotic regimens regulated under the supervision of an infectious disease specialist. After initial and subsequent debridement of the open fractures, final fixation with a nail was performed when the attending surgeon and infectious diseases specialist were in agreement regarding the sterilization of the wound. All patients were operated on a traction table using standard techniques. An additional stoppa incision was made on the fracture site for the reduction of the fracture if closed reduction of the fracture could not be obtained. The size and locking modality employed was at the discretion of the operating surgeon at the time of the surgery.
Patients were allowed to attempt full weight bearing at postoperative day 1 with crutches or walkers if an anatomic reduction could be obtained during surgery. However, if there was any doubt regarding the stability of the fixation, particularly in elderly patients with infra-isthmal fractures and in patients with unstable supra-isthmal fractures, patients were allowed to attempt toe-touch walking with the assistance of a walker until callus was seen on X-rays during follow ups. Quadriceps strengthening exercises were started at postoperative day 1 and were continued until pain free full knee flexion and straight leg raise were obtained. Clinical and radiographic assessments of the progress of healing and complications were carried out during all visits. Follow-up visits were conducted at 3 weeks, 6 weeks, 3 months, 4 months, 6 months, and 1 year. If there was a detectable problem, the patient was advised to come in for follow-up earlier than scheduled.

\section{Statistical Analyses}

All statistical analyses were performed using SPSS 25.0 (IBM Corporation, Armonk, New York, United States) and PAST3 (Hammer, Ø., Harper, D.A.T., Ryan, P.D. 2001. Paleontological statistics). For quantitative data, independent samples $T$ tests were used together with bootstrap results while Mann-Whitney $U$ test was used with Monte Carlo simulation technique in the comparison of two independent groups. For the comparison of categorical variables, Pearson-Chi-Square, exact, and Monte Carlo simulation results were tested with Fisher exact test, exact results with Fisher-Freeman-Holton test, and Monte Carlo simulation technique. Column ratios were compared to each other followed by Benjamini-Hochberg correction and expressed as $p$ values. Odds ratios with $95 \%$ confidence intervals (CI) were used where appropriate. Quantitative variables are expressed as mean \pm standard deviation or as medians; categorical variables are given as numbers with percentages with $95 \%$ CIs. p values $<0.05$ were considered statistically significant.

\section{RESULTS}

After primary review of the records, 163 patients were identified who had undergone operations for femoral shaft fractures from 2010-2018. Of these patients, 77 were excluded based on inclusion criteria, resulting in 86 patients (90 fractures) included in the study. A total of 


\begin{tabular}{|c|c|c|c|}
\hline $\begin{array}{l}\text { Patients and } \\
\text { fractures characteristics }\end{array}$ & $\begin{array}{l}\text { T. Fossa Entrance } \\
\qquad \begin{array}{l}(n=67) \\
(n=65)\end{array} \\
\text { Median (min./max.) }\end{array}$ & $\begin{array}{l}\text { G. Trochanter Entrance } \\
\qquad \begin{array}{l}(\mathrm{n}=23) \\
(\mathrm{n}=21)\end{array} \\
\text { Median (min./max.) }\end{array}$ & $\mathrm{p}$ \\
\hline \multirow[t]{2}{*}{ Age } & $34(16 / 60)$ & $50(18 / 60)$ & $0.308^{\mathrm{u}}$ \\
\hline & $\%$ & $\%$ & \\
\hline \multicolumn{4}{|l|}{ Gender } \\
\hline Female & 29.2 & 42.9 & $0.289^{\text {pe }}$ \\
\hline Male & 70.8 & 57.1 & \\
\hline \multicolumn{4}{|l|}{ Trauma pattern } \\
\hline Low energy & 37.3 & 52.2 & $0.229^{p e}$ \\
\hline High energy & 62.7 & 47.8 & \\
\hline \multicolumn{4}{|l|}{ OTA/AO class } \\
\hline $32 \mathrm{~A}$ & 61.2 & 56.5 & $0.423^{f f}$ \\
\hline $32 B$ & 22.4 & 34.8 & \\
\hline $32 \mathrm{C}$ & 16.4 & 8.7 & \\
\hline \multicolumn{4}{|l|}{ Level of fracture } \\
\hline Supra-isthmal & 59.7 & 39.1 & $0.170^{\mathrm{pm}}$ \\
\hline Isthmal & 22.4 & 26.1 & \\
\hline Infra-isthmal & 17.9 & 34.8 & \\
\hline Open fracture & 16.4 & 4.3 & $0.284^{f}$ \\
\hline \multicolumn{4}{|l|}{ ASA } \\
\hline I & 70.8 & 52.4 & $0.113^{f f}$ \\
\hline II & 24.6 & 47.6 & \\
\hline III & 4.6 & 0.0 & \\
\hline Accompanying fracture & 14.9 & 17.4 & $0.748^{f}$ \\
\hline
\end{tabular}

min.: Minimum; max.: Maximum; u: Mann Whitney u test (Monte Carlo); p: Pearson Chi Square Test (e Exact, m Monte Carlo); f: Fisher Exact Test (Exact); ff: Fisher Freeman Halton Test (Monte Carlo).

65 patients (67 fractures) were operated on using TFE nails, and 21 patients ( 23 fractures) were operated on using GTE nails. There were two bilateral fractures in the TFE group and two in the GTE group.

Preoperative patient characteristics, including age, sex, ASA, AO/OTA class, level of fracture, accompanying fractures, open fractures, and trauma pattern were similar between groups (Table 1). The median follow-up times were 14 months (12-74 months) for the TFE group and 15 months (12-60 months) for the GTE group. Duration of hospital stay after surgery was significantly longer in the TFE group (median 7 days; range 2-20) compared to the GTE group (median 4 days; rage 3-15 days). There were no differences between groups in terms of duration of surgery, decrease in Hgb levels after surgery, and need for transfusion after surgery ( $p>0.05$; Table 2$)$.

Proximal and distal locking features of the patients were similar for both groups $(\mathrm{p}=0.128)$. Time to union was similar for both groups with a median of 16 weeks (range 8-32 weeks) for the GTE group and 20 weeks (range 8-48 weeks) for the TFE group ( $p=0.166$; Table $2)$. Delayed union was detected in 18 patients in the TFE group compared to 8 patients in the GTE group $(p>0.05)$. Rate of non-union and additional surgeries performed were similar for both groups $(p>0.05)$. Three additional surgeries ( 3 patients) were performed in the GTE group: one was for removal of the proximal locking screws after union causing trochanteric irritation, one was for removal of all implants at the request of the 


\begin{tabular}{|c|c|c|c|}
\hline $\begin{array}{l}\text { Groups } \\
\text { Fractures } \\
\text { Patients }\end{array}$ & $\begin{array}{l}\text { T. Fossa Entrance } \\
\qquad(n=67) \\
\quad(n=65) \\
\text { Median (min./max.) }\end{array}$ & $\begin{array}{l}\text { G. Trochanter Entrance } \\
\qquad \begin{array}{l}(n=23) \\
(n=21)\end{array} \\
\text { Median (min./max.) }\end{array}$ & $\mathrm{p}$ \\
\hline Follow up time (months) & $14(12 / 74)$ & $15(12 / 60)$ & $0.622^{u}$ \\
\hline Post-operative stay in hospital (days) & $7(2 / 20)$ & $4(3 / 15)$ & $0.002^{u}$ \\
\hline \multirow[t]{2}{*}{ Time to union (weeks) } & $20(8 / 48)$ & $16(8 / 32)$ & $0.166^{u}$ \\
\hline & Mean \pm SD & Mean \pm SD & \\
\hline Decrease in HGB levels (unites) & $2.75 \pm 1.53$ & $2.35 \pm 1.03$ & $0.249^{\text {ra }}$ \\
\hline \multirow[t]{2}{*}{ Duration of surgery (minutes) } & $79 \pm 19.7$ & $71 \pm 20.3$ & $0.890^{4}$ \\
\hline & $\%$ & $\%$ & \\
\hline Post-operative blood transfusion & 10.4 & 13.0 & $0.712^{f}$ \\
\hline \multicolumn{4}{|l|}{ Reduction technique } \\
\hline Closed reduction & 52.2 & $82.6^{\mathrm{A}}$ & $0.013^{p}$ \\
\hline Open reduction & $47.8^{\mathrm{B}}$ & 17.4 & $4.34(1.33-14.13)^{\mathrm{OR}}$ \\
\hline \multicolumn{4}{|l|}{ Proximal locking mode } \\
\hline 1 screw to collum & 3.0 & 8.7 & \\
\hline 2 screws to collum & 25.4 & 39.1 & $0.128^{\mathrm{ff}}$ \\
\hline 1 screws to Lesser troc. & 71.6 & 52.2 & \\
\hline Loss of reduction during follow-ups & 4.5 & 4.3 & $0.999^{f}$ \\
\hline Malreduction at initial postoperative stage & 3 & 12.9 & $0.132^{\mathrm{ff}}$ \\
\hline
\end{tabular}

min.: Minimum; max.: Maximum; u: Mann Whitney u test (Monte Carlo); p: Pearson Chi Square Test (e Exact, m Monte Carlo); f: Fisher Exact Test (Exact); ff: Fisher Freeman Halton Test (Monte Carlo).

patient after union, and one was a revision of the fixation with a plate and nail exchange because of nonunion after 10 months. This patient achieved union after 1 year of revision surgery. Five additional surgeries (4 patients) were performed in the TFE group, of which one was an exchange nail surgery due to nonunion 1 year after primary surgery for an isthmal fracture. This patient achieved union at postoperative 8 months after revision surgery. The next case was for removal of the proximal locking screw after union due to irritation of the tensor fascia lata. The final two were removal of the distal static screw at postoperative months 6 and 11 due to nonunion. These patients achieved union at postoperative months 12 and 16, respectively. All implants in the latter patient were removed at postoperative year 4 at the request of the patient.

Malreduction and iatrogenic fractures were more common in the GTE group for treatment of supra-isthmal fractures $(p<0.05)$. No malreduction or loss of re- duction was detected among supra-isthmal fractures in the TFE group. In addition, iatrogenic fracture of the collum femoris was not detected in TFE group; however, a varus malreduction was detected in three patients with supra-isthmal fractures in the GTE group. Iatrogenic fractures of the lateral and iatrogenic walls of the proximal fragment were detected in two patients. No further intervention was needed for two patients exhibiting a varus between 10 to 15 degrees, as these patients had achieved complete union; however, the last patient did undergo revision of the fixation with a plate and nail exchange because of nonunion (Fig. 1, 2).

For both groups all isthmal fractures were reduced in an anatomic manner and no loss of reduction was detected among these patients $(p>0.05)$. Initial postoperative malreduction was detected for two patients among infra-isthmal fractures in the TFE group, with both patients achieving union without further deterioration of the reduction. However, loss of anatomic reduction was 

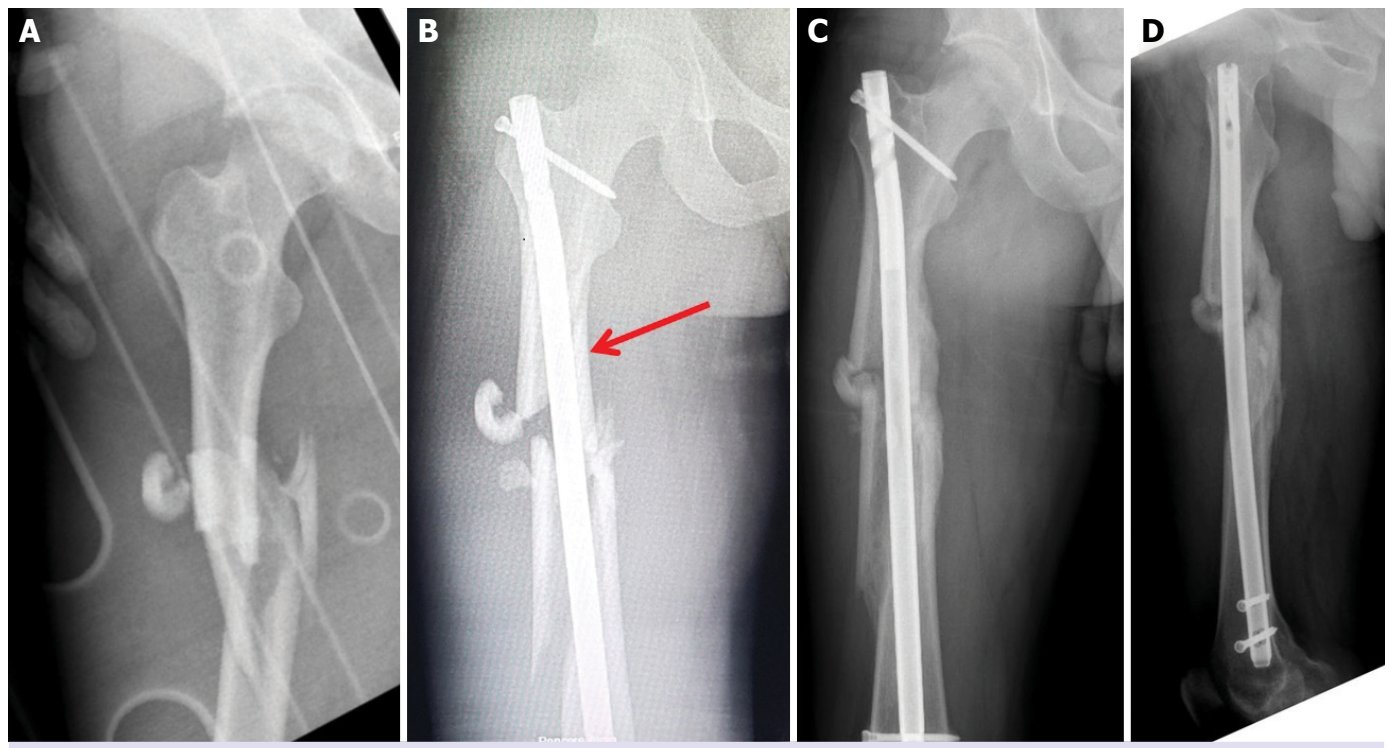

FIGURE 1. (A) A 24-year-old man sustained an AO type 32 c3.1 fracture after a motor vehicle accident. (B) The patient was operated on using a GTE nail. A red arrow shows an iatrogenic distal medial cortex fracture and with $16^{\circ}$ of varus malalignment. (C, D) X-rays taken at postoperative 13 months shows union of the fracture in varus mal-alignment.
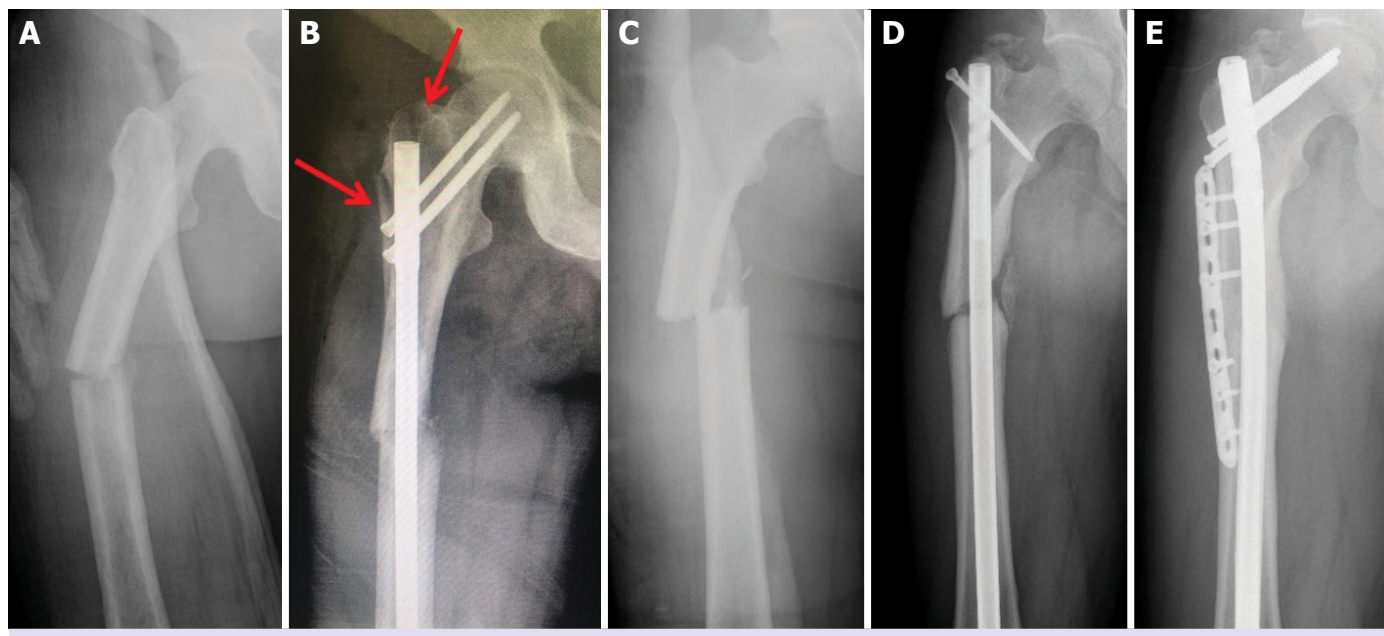

FIGURE2. (A) A 55-year-old woman sustained an AO type 32 A3.1 fracture. (B) The patient was operated on using a GTE nail. Red arrows show iatrogenic communition of the postero-lateral cortex arising from inappropriate positioning of the entrance point. (C) A 48-year-old man sustained an AO type 32 A2.1 fracture. (D) The patient was operated on using a GTE nail. An $X$-ray taken at postoperative 10 months revealed nonunion with $14^{\circ}$ of varus malalignment. (E) A revision operation with nail exchange and additional fixation with plates was carried out at postoperative 11 months. X-rays taken at postoperative 24 months showed a perfect union and alignment of the fracture with corrected varus malalignment.

detected in three patients presenting with infra-isthmal fractures. Of these patients, two developed a recurvatum displacement of the distal fragment (10 and 15 degrees, respectively) and one patient developed a 10-degree varus displacement of the distal fragment. Three patients achieved full union, after which no further intervention was required. Initial postoperative reduction was detected to be anatomic for all patients with infra-isthmal fractures in the GTE group. Loss of anatomic reduction was detected in one patient with an infra-isthmal frac- 


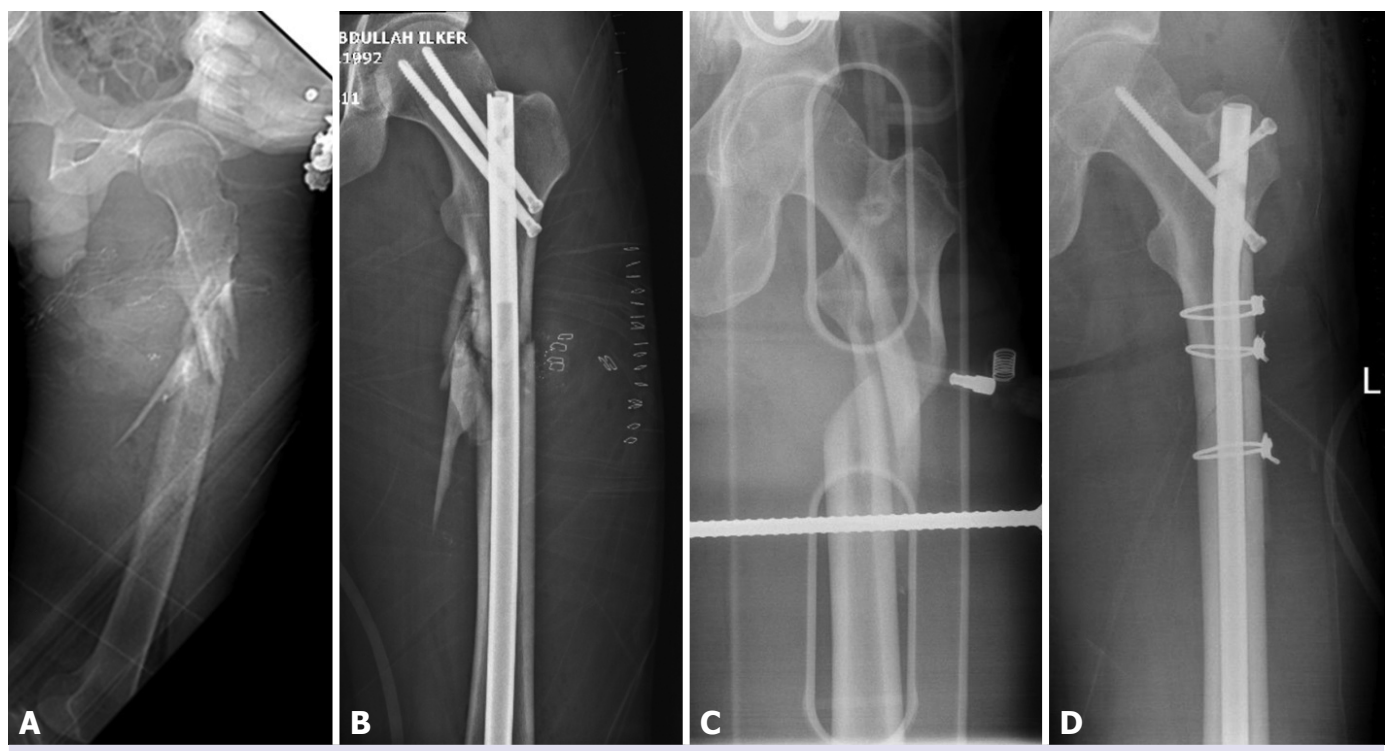

FIGURE 3. (A) A 24-year-old man sustained an AO type 32 c3.1 fracture as the result of a gunshot injury. (B) An open reduction and fixation with a TFE nail was performed. Early postoperative X-rays revealed excellent realignment. (C) A 44-year-old man sustained an AO type 32 c1.1 fracture after a fall from $10 \mathrm{~m}$. (D) An open reduction and fixation with cables and a GTE nail was performed. Early postoperative $\mathrm{X}$-ray revealed excellent realignment.

ture. This patient developed a 15 -degree recurvatum displacement of the distal fragment; however, no further intervention was needed was for this particular patient as union was achieved without further deterioration of the distal fragment $(p=0.132)$.

There was a significantly higher need for open reduction via a small stop incision in the TFE group $(p=0.013$; Table 2$)$. An open reduction was performed in 32 fractures in the TFE group compared to only 4 fractures in the GTE group. Of the affected patients in the TFE group, 4 were $\mathrm{AO} 32 \mathrm{C}$ isthmal fractures for which the stoppa incisions were expanded and additional fixation of the fracture with cables were carried out. By contrast, only for one supra-isthmal fracture required additional fixation with an expanded incision in the GTE group (Fig. 3).

There were no differences between the groups in terms of infection incidence or other related complications, such as deep vein thrombosis (DVT; $p>0.05$ ). There were no deep infections in either group; however, there were six incisional infections in the TFE group and three in the GTE group during the early postoperative stage, all of which were remediated with irrigation and debridement along with oral antibiotics. A survey for DVT was carried out only in patients complaining of symptoms consistent with a potential diagnosis. Four patients in the TFE group and three in the GTE group were found to have DVT during the follow-up period, at which point all patients received the appropriate treatment under supervision of a vascular surgeon. No further complications were detected in these patients.

\section{DISCUSSION}

There is an ongoing debate as to the best entrance option for femoral shaft fractures. Several studies have reported on different clinical and radiological factors that could be affected by different entrance points. Ricci et al. [1, 7] found the use of a GTE point to decrease both overall surgery time and fluoroscopy time. Similarly, $\mathrm{Ha}$ et al. [9] reported decreased operation time and less blood loss in GTE procedures, particularly in overweight patients. However, several papers have also reported significantly more iatrogenic fractures in the GTE group. Stannard et al. [3] reported intraoperative parameters including fluoroscopy time and duration of surgery to be better in the GTE group with no differences in functional outcomes. Although some cadaveric studies have claimed that the GT entrance may decrease morbidity due to a reduction in soft tissue injury, [5] other clinical studies have shown that functional outcomes, particularly in regard to the gluteus medius, abductor muscles, and tensor fasciae to 
be similar between different entrance points [3]. However, only a handful of studies have directly compared the effects of the entrance point among supra-isthmal, isthmal, and infra-isthmal femoral shaft fractures [9]. In our study, we evaluated the effects of the different entrance points in these fractures at different levels. Our results revealed that iatrogenic collum femoris fractures are less common than anticipated with use of TFE nails, while iatrogenic fractures are more common with GTE nails. Furthermore, although infra-isthmal and isthmal fractures may be readily operated on using either nail, TFE nails may offer better outcomes in patients with supra-isthmal fractures. Moreover, we found that the duration of surgery, the need for transfusion, and the amount of blood loss were all similar between groups, in contrast to previous studies. However, the duration of postoperative stay in the hospital was longer in the TFE group, likely due to the higher number of open reductions performed in this group.

Although several authors have recommended the use of a more lateralized entrance for treatment of femoral shaft fractures, $[1,5,12]$, further studies have revealed that despite developments in the design of GTE nails, more iatrogenic fractures with associated varus malalignment can be anticipated [13]. However, previous studies had not classified these fractures according to the level at which they occurred, which means it is not obvious whether these iatrogenic fractures would be evenly distributed across all fractures or would be isolated to a particular group. In this study, complications rates, union time, and other parameters were similar between groups for isthmal and infra-isthmal fractures, although more iatrogenic fractures were detected in the GTE group in patients with supra-isthmal fractures. Diab et al. [14] reported acceptable complication rates with a GTE nail in treatment of subtrochanteric fractures and had concluded that a start site lateral to the tip of the greater trochanter may be associated with varus malreduction.

A review of the current literature reveals that a meticulous assessment of supra-isthmal fractures must be carried out before deciding on a surgical entrance point, but supports the use of either entrance after meticulous assessment of the fracture [15]. Indeed, there is no "right" or "wrong" entry portal when contemplating nailing supra-isthmal femoral fractures. When choosing the appropriate starting site for these injuries, the surgeon should consider many potential factors, all of which contribute to appropriate portal location. Fully understanding all subtleties related to each particular starting point will help the surgeon individualize the entry portal for each fracture [16]. In this study, supraisthmal fractures constituted the majority of the TFE group, with open reductions performed in 28 of 40 supraisthmal fractures in this group. By contrast, only one open reduction was performed among the 9 supraisthmal fractures in the GTE group. In the case of supraisthmal fractures, if an appropriate reduction with traction prior to nail entrance cannot be obtained, an open reduction followed by reduction with clamps or additional cables will ease the introduction of the nail and decrease the risk for an iatrogenic fracture [16]. In this study, no iatrogenic fractures were detected among isthmal and infra-isthmal fractures indicating that an intact long proximal segment prevents iatrogenic communition at the entrance point. This shows that by creating a relatively complete medullary canal through an open reduction prior to the nail insertion for supraisthmal fractures, we were able to decrease the risk for fracture at the entrance point. Although the higher number of open reductions performed in the TFE group may have prolonged the duration of hospital stays in this group, it may account for the lower level of complications seen in the TFE group.

Another factor that must be assessed among different entrance points is that future conversion to a total hip arthroplasty (THA) may be more difficult in patients treated using a GTE nail, although no direct assessment has been performed to date. Bercik et al. [17] compared the use of lateral plating and GTE IM nailing in fracture patients and found that both operative times and blood loss were significantly greater in GTE patients during conversion to THA.

Although the clinical importance of a varus malalignment in patients treated with GTE nails remains unclear, a higher re-operation rate due to varus malalignment in these patients have been reported [14]. For any varus malalignment, the mechanical axis would be shifted medially on the knee resulting in an increased stress on the medial knee compartment. However, no studies have directly assessed this issue in either GTE or TFE patients. Our patients with varus malalignment underwent their operations 14-16 months prior to correction, after which neither knee pain nor restricted range of motion were detected during the relatively short follow-up time.

For supra-isthmal fractures, nails create increased stress at two points: the proximal lateral cortex and the distal antero-medial cortex of the proximal fragment. During nail insertion, the tip leans toward the distal ante- 
ro-medial cortex of the proximal fragment; the entrance point serves as a hinge and further insertion increases the stress at that those points. If the entrance point is not wide enough or positioned too laterally, anteriorly, or posteriorly, this will weaken the cortex at this level [13]. With GTE nails, the distal antero-medial cortex of the proximal fragment tends to experience excessive wear as the lateral entrance pushes the guide wire medially, which creates a wider medullary canal after reaming at distal proximal fragment. However, a thinner and wider medullary canal at the distal antero-medial cortex of proximal fragment can cause varus malalignment and iatrogenic fractures. To prevent varus malalignment, a nail that fills the medullary canal must be used; however, a larger nail means more reaming and thinning of the antero-medial cortex, combined with greater stress on the on medial cortex during the nail entrance. As seen in our study, the nail diameter must fill the medullar canal at the most distal level of proximal fragment (Fig. 3). In cases where this was not achieved, the proximal fragment will be pulled anteriorly and laterally by muscles, which creates a varus malalignment, particularly in cases of GTE nails (Fig. 1, 2). By contrast, TFE nails offer an entrance point in line with the medullary canal, allowing even nails with a diameter smaller than the medullary canal of the proximal fragment to be used with less risk for varus malalignment (Fig. 1A, B). Furthermore, this straight trajectory for TFE nails prevents unnecessary reaming of the distal medial cortex of the proximal fragment and increased stress at the point of entry [18]. The major concern with the use of TFE nails is the risk for iatrogenic collum femoris fracture; however, no such fractures were detected in this study. We believe that a cautious positioning of the entrance point prevents iatrogenic neck fractures.

Proximal locking mode of the supra-isthmal fractures also affects the stability of the fixation. Conventional reconstruction nails allow two parallel screws to be placed in the femoral head. However, for bones exhibiting overly narrow necks or in cases of inappropriately positioned (superior or inferior) nails, placing two screws into the neck may be very difficult [19]. We encountered this kind of situation in two cases in the TFE group as well as two cases in the GTE group. However, none of these patients were given restrictions in terms of early weight bearing, and deterioration of the fixation was not detected in any patient (Fig. 3). In such cases we now use a short locking nail introduced from the hole aiming to the lesser trochanter, as recommended by Nicalaou et al. [16].
Postoperative malalignment in infra-isthmal fractures for both groups were similar, and no further intervention was needed for any of the patients. These kinds of fractures are prone to malalignment as there is a discrepancy in the width of the medullary canal at this level. Using Poller screws or after proper reduction via locking the nail in at least two planes can prevent initial malreduction and further deterioration of the reduction. Also using a retrograde intramedullary nail specifically designed for distal oblique fractures can prevent this complication [20].

Taken together, the data presented here show that nails inserted via a trochanteric entrance or trochanteric fossa entrance were safe and efficient for the treatment of isthmal and infra-isthmal fractures. However, varus malalignment associated with iatrogenic fractures was more common with trochanteric entrance nails. Fixation of supra-isthmal fractures with GTE nails is more demanding. Our results concluded that TFE nails may be a safer option for surgical treatment of supra-isthmal fractures.

The major limitation of our study was that it was not a prospective randomized study, and intraoperative fluoroscopy times were not recorded. Furthermore, a functional assessment could not be carried out, body mass indexes were not assessed, and the overall number of patients was too small to support more definitive conclusions.

Ethics Committee Approval: The Umraniye Training and Research Hospital Clinical Research Ethics Committee granted approval for this study (date: 17.04.2019, number: B.10.1.TKH.4.34.H.GP.0.01/77).

Conflict of Interest: No conflict of interest was declared by the authors.

Financial Disclosure: The authors declared that this study has received no financial support.

Authorship Contributions: Concept - MSS; Design - MSS; Supervision - MSS, TTO; Fundings - TTO; Materials - TTO; Data collection and/or processing - MSS, TTO; Analysis and/or interpretation - MSS, TTO; Literature review - MSS; Writing - MSS, TTO; Critical review - MSS, TTO.

\section{REFERENCES}

1. Ricci WM, Devinney S, Haidukewych G, Herscovici D, Sanders R. Trochanteric nail insertion for the treatment of femoral shaft fractures. J Orthop Trauma 2005;19:511-7. [CrossRef]

2. Winquist RA, Hansen ST Jr, Clawson DK. Closed intramedullary nailing of femoral fractures. A report of five hundred and twenty cases. J Bone Joint Surg Am 1984;66:529-39. [CrossRef]

3. Stannard JP, Bankston L, Futch LA, McGwin G, Volgas DA. Functional outcome following intramedullary nailing of the femur: a prospective 
randomized comparison of piriformis fossa and greater trochanteric entry portals. J Bone Joint Surg Am 2011;93:1385-91. [CrossRef]

4. Sheth U, Gohal C, Chahal J, Nauth A, Dwyer T. Comparing Entry Points for Antegrade Nailing of Femoral Shaft Fractures. Orthopedics 2016;39:e43-50. [CrossRef]

5. Ansari Moein CM, Verhofstad MH, Bleys RL, van der Werken C. Soft tissue anatomy around the hip and its implications for choice of entry point in antegrade femoral nailing. Clin Anat 2008;21:568-74. [CrossRef]

6. Schwieger K, Moein CA, Bösl O, Verhofstad M. Lateral insertion points in antegrade femoral nailing and their influence on femoral bone strains. AO Dev News 2007;1:48-9.

7. Ricci WM, Schwappach J, Tucker M, Coupe K, Brandt A, Sanders R, et al. Trochanteric versus piriformis entry portal for the treatment of femoral shaft fractures. J Orthop Trauma 2006;20:663-7. [CrossRef]

8. Tucker MC, Schwappach JR, Leighton RK, Coupe K, Ricci WM. Results of femoral intramedullary nailing in patients who are obese versus those who are not obese: a prospective multicenter comparison study. J Orthop Trauma 2007;21:523-9. [CrossRef]

9. Ha SH, Kim WH, Lee GC. Results of intramedullary nailing of femoral shaft fracturetrochanteric entry portal (Sirus nail) versus piriformis entry portal (M/DN nail). J Korean Fract Soc 2014;27:50-7. [CrossRef]

10. Meinberg EG, Agel J, Roberts CS, Karam MD, Kellam JF. Fracture and Dislocation Classification Compendium-2018. J Orthop Trauma 2018;32 Suppl 1:S1-170. [CrossRef]

11. Gustilo RB, Anderson JT. Prevention of infection in the treatment of one thousand and twenty-five open fractures of long bones: retrospective and prospective analyses. J Bone Joint Surg Am 1976;58:453-8.

12. Linke B, Ansari Moein C, Bösl O, Verhofstad MH, van der Werken $\mathrm{C}$, Schwieger $\mathrm{K}$, et al. Lateral insertion points in antegrade femoral nailing and their influence on femoral bone strains. J Orthop Trauma 2008;22:716-22. [CrossRef]

13. Prasarn ML, Cattaneo MD, Achor T, Ahn J, Klinger CE, Helfet DL, et al. The effect of entry point on malalignment and iatrogenic fracture with the Synthes lateral entry femoral nail. J Orthop Trauma 2010;24:224-9. [CrossRef]

14. Mustafa Diab M, Wu HH, Eliezer E, Haonga B, Morshed S, Shearer DW. The impact of antegrade intramedullary nailing start site using the SIGN nail in proximal femoral fractures: A prospective cohort study. Injury 2018;49:323-7. [CrossRef]

15. Jackson C, Tanios M, Ebraheim N. Management of Subtrochanteric Proximal Femur Fractures: A Review of Recent Literature. Adv Orthop 2018;2018:1326701. [CrossRef]

16. Nicolaou D, Watson JT. Nailing proximal femur fractures: how to choose starting point and proximal screw configuration. J Orthop Trauma 2015;29 Suppl 4:S22-7. [CrossRef]

17. Bercik MJ, Miller AG, Muffly M, Parvizi J, Orozco F, Ong A. Conversion total hip arthroplasty: a reason not to use cephalomedullary nails. J Arthroplasty 2012;27:117-21. [CrossRef]

18. Gausepohl T, Pennig D, Koebke J, Harnoss S. Antegrade femoral nailing: an anatomical determination of the correct entry point. Injury 2002;33:701-5. [CrossRef]

19. Pugh KJ, Morgan RA, Gorczyca JT, Pienkowski D. A mechanical comparison of subtrochanteric femur fracture fixation. J Orthop Trauma 1998;12:324-9. [CrossRef]

20. Poyanl OS, Soylemez MS, Ozkut AT, Esenkaya I, Unal OK, Kılıncoglu V. GUse of provisional K wires instead of Poller screws for treatment of diametaphyseal fractures of the distal femur and proximal and distal tibia. Acta Orthop Belg. 2016;82:579-85. 